\title{
How Reliable is Your Covid 19 Tests? Need to Validate and Verify Covid 19 Tests in the Laboratory Before Clinical Use
}

\author{
Amit Pruthi ${ }^{1}$, Maneesh Baghi ${ }^{2}$, Ashok Rattan ${ }^{3 *}$ and Sanjeev Vashishta ${ }^{4}$ \\ ${ }^{1}$ Head Corporate Quality, Pathkind Labs, Gurgaon, India \\ ${ }^{2}$ Director, Regional Reference Laboratory, Pathkind Labs, Gurgaon, India \\ ${ }^{3}$ Advisor, Pathkind Labs, Gurgaon, India \\ ${ }^{4}$ Managing Director and Chief Executive Officer, Pathkind Labs, Gurgaon, India \\ *Corresponding Author: Ashok Rattan, Professor, Advisor, Pathkind Labs, Gurgaon, \\ India.
}

Received: July 14, 2020

Published: September 26, 2020

(C) All rights are reserved by Ashok

Rattan., et al

\begin{abstract}
On the last day of last year, China informed World Health Organisation was a cluster of pneumonia cases in Wuhan, caused by a novel coronavirus, which was identified as SARS CoV 2 based on sequences of viral agent available by $10^{\text {th }}$ January 2020 and RT PCR tests developed to identify this RNA virus. The disease caused by this virus was labelled as Covid 19. The rapid spread of this virus from China to all parts of the world including India, led this outbreak to be labelled as a pandemic.

In January 2020, India had only one laboratory testing for Covid 19, at the Indian Council of Medical Research's National Institute of Virology, Pune. In Mid September, the testing facilities had been ramped upto 1700 laboratories, both in the government and private set up, across the country, performing molecular tests for diagnosis of Covid 19 - an unparallel achievement in the history of Indian health system.

In the absence of an effective treatment, prevention is the best strategy, which resolves around testing. RT PCR for Covid 19 involves multiple complex steps. This paper describes the minimal steps each performing laboratories must take to ensure quality in their testing. It is essential that testing laboratory verify manufacturer's claim and perform ongoing monitoring of tests to ensure that reliable and reproducible results are available to decision makers to make evidence based informed decisions for containment of this infection. While EQAS has been announced, but no sample has yet been circulated. Interlaboratory comparison should be encouraged between ICMR designated Reference laboratories and other performing laboratories.
\end{abstract}

Keywords: Severe Acute Respiratory Syndrome (SARS); Covid 19; ICMR

\section{Introduction}

On $31^{\text {st }}$ December 2019, the World Health Organization was informed about a cluster of pneumonia cases in Wuhan, China. Subsequent investigations identified a novel coronavirus that closely resembled the virus of severe acute respiratory syndrome (SARS) which had occurred in 2003. Person to person transmission among family contacts and health care workers were reported. Infection appeared to be spread by the travellers from Wuhan in the other parts of the country and world. As with SARS and MERS control, highly sensitive and specific laboratory diagnosis of this novel coronavirus was essential for case identification, contact tracing, animal source finding and infection control [1]. By $10^{\text {th }}$ January 2020 , complete sequence of one virus was available in the public domain to which five more sequences were added the next day. The availability of these sequences early in the epidemic facilitated the development of specific primers. The first Real Time PCR for this RNA virus targeted RNA dependent RNA polymerase (RdRp), envelope and nucleocapsid $(\mathrm{N})$ genes was published on $23^{\text {rd }}$ January 
2020. Corona virus can grow in Vero cell lines, but because of the high transmissibility and pathogenic potential as well as slow turn around time and requirement of Biosafety III facilities, this was not a preferred method for clinical diagnosis. Serology tests for detection of antigen or antibodies were not found suitable, either because of sensitivity or specificity for the purpose of diagnosis followed by containment of this infection, which by now was called Covid 19 infection and the virus termed as SARS CoV 2. CDC Atlanta developed a RT PCR focused on N gene wherein they identified three regions, named N1, N2 and N3. This test did not perform well when transferred to other public health laboratories and the Koch Institute's RT PCR was adopted by WHO for use. Initially it was two step process, but the rapid spread of this virus has led to the development of single tube, single step multiplex RT PCRs for rapid and definitive diagnosis of the infection [2].

In March 2020, only one laboratory was performing this test, which has been rapidly under the leadership and guidance provided by ICMR to over 1700 labs in all parts of the country [3]. In this, NABL also played a very positive role by either allowing accredited labs to add RT PCR for RNA Virus to the scope or performing full accreditation during the lock down period by video conferencing. ICMR dictated that all private labs must be NABL accredited and should have RT PCR for RNA in their scope of accreditation. This prerequisite was, however, not applied to the government owned laboratories as though if your salary was paid by the tax payer, you would magically acquire unimpeachable quality properties, rest of course, would have to bring validation data! A test must be shown to be fit for purpose so that users can have confidence in the results produced by its application. Method validation and verification provide objective evidence that a method is fit for purpose.

All private laboratories permitted to test for covid 19 are therefore accredited by NABL against ISO 15189:2012 standards which requires all accredited labs to verify and validate all tests before use as per clause 5.5.1.1. and 5.5.1.3 [4].

Under 5.5.1.1. Validated examination procedures used without modification must be subject to independent verification by the laboratory prior to routine use. Information is to be obtained from the manufacturer regarding the performance characteristics of the procedure. The verification must confirm that the performance claims for the procedure have been met. The procedure used for verification must also be documented, with the obtained results recorded. Verification results are to be reviewed by the appropriate staff.

Under 5.5.1.3. greater detail regarding the situations when validation of examination procedures is required has been included in this clause. Validation must be performed for non-standard methods, laboratory developed methods, standard methods used outside their intended scope and validated methods subsequently modified. But how has not been described.

It is established principles in all accredited laboratories that the laboratory is required to verify certain performance characteristics of the test. For qualitative tests this includes comparison of positive and negative test results to a comparable test method. Specimens for the verification can include external control material, cultured organisms or proficiency testing material but must include positive and negative patients samples. For quantitative tests, the manufacturer's limit of detection, linearity, reportable range and precision should be verified by the laboratory, as well as a comparison of patient test results across the reportable range of the test. Specimens for the verification can include quantitative external control material, cultured organisms (quantified) and proficiency testing material but must include patient samples too [5].

Verification is a one time process performed to determine or confirm a test's expected performance prior to implementation in the clinical laboratory (simply put, it addresses the question, "Does the test work?"), validation on the other hand is an ongoing process of monitoring a test to ensure that it continuously performs as expected, ("it addresses the question, "Does the test still work?").

Verification confirms that test performs as per manufacture's specification and it should be carried out A. When introducing a new test, B. A new process or C. From a different manufacturer. This is carried out by performing several analysis using a panel of atleast 20 specimens. With a well-defined panel, these analysis can be completed in a couple of days. What needs to be done by the laboratory is:

1. Accuracy (sensitivity and specificity)

2. Reproducibility

3. Reportable range

4. Reference range

5. Other tests characteristics, such as linearity, specimen stability or carryover when applicable. 
Individual laboratories can determine Accuracy by using atleast 20 specimens (containing both positive and negative samples) and must be more than $90 \%$ in agreement with the reference method. Manufacturers have determined analytical sensitivity by using synthetic SARS CoV 2 RNA after spiking into negative respiratory clinical matrices (NP swab and BAL), a LOD of 6.25 genomic copies/ul for NP swabs and $12.5 \mathrm{cp} / \mathrm{ul}$ for BAL has been reported. Analytical specificity has been determined by the manufacturers to explore the cross reactivity of COVID 19 RT PCR test both by in silico analysis and by testing whole organisms or purified nucleic acid from a panel of organisms usually found in the respiratory tract. BLAST analysis has shown no homology with primers and probes used for COVID 19 RT PCR and individual laboratories needed not produce any further evidence to substantiate this.

Individual laboratories can confirm reproducibility by using several samples from the 20 sample panel running them in duplicate, repeat second run with a second operator. If same or comparable results are obtained, reproducibility is good. Reportable range is determined by testing positive samples with low and high value. The test should detect both weak and strong positives. Published Reference range could be used. Or can use negative samples from the panel to determine if normal is negative. Manufacturers should have already performed clinical evaluation using either natural or contrived clinical samples. A total of 100 individual clinical respiratory samples, $50 \mathrm{NP}$ swabs and $50 \mathrm{BAL}$ as negatives and atleast 80 either natural or contrived positives. RT PCR should show $100 \%$ agreement with positive and negative expected results.

In view of the ongoing pandemic, USA FDA has suggested that accredited laboratories who decide to use EUA kits from authorized manufacturers can verify test performance to perform testing in their own laboratories [6]. Before that, each testing laboratory must ensure that:

1. Personnel are trained and qualified to perform testing based on the specific level of authorization received.

2. Follow the assay or test system's protocol without modification.

3. Verify both positive and negative samples in the study.

4. Perform IQC at least as frequently as stated in the manufacturer's package insert.

If, however, laboratory has developed its own test or has modified an approved test, then each laboratory must validate the test.
Validation studies can be divided into comparative and primary validation.

\section{Comparative validation}

Comparative (i.e. correlation or cross) validation is usually applied to bioanalytical methods and aims to demonstrate equivalent performance between two (or more) methods used to generate data within the same study by comparing the validation parameters. There is no single test of establishing method equivalence or numerical acceptance criteria for it. Generally, a method with the greatest sensitivity or highest recovery for the target analyte is the best. To determine if the alternative method mean is not statistically different from the reference method mean, a one way analysis of variance or a paired t-test by sample type and analyte concentration is performed. Comparative validation studies of qualitative methods involve the identification of operating characteristics of the method (e.g. sensitivity, selectivity, presumptive false positive and presumptive false negative).

\section{Primary validation}

For situations where comparative validation is not applicable (e.g. in-house-developed methods, standard methods that have been modified in such a way that the final result could be influenced, standard methods used outside the intended scope, use of an alternative isolation or detection principle, as well as rapid methods), primary validation must be undertaken prior to introducing the method. In such cases validation becomes an exploratory process with the aim of establishing operational limits and performance characteristics of the alternative, new or otherwise inadequately characterised method. It should result in numerical and/or descriptive specifications for the performance of the method.

So validation performance characteristic that should be determined prior to reporting patient results include analytical and diagnostic sensitivity and analytical and diagnostic specificity, precision, linearity (for quantitative tests), reportable range of patient test results; the reference range (normal value); performance with clinical specimens and any other applicable performance characteristic.

Analytical sensitivity refers to the ability of a test to detect a given analyte (i.e. lower limit of detection). Analytic specificity refers to the degree to which related organisms are not detected by a test. Precision refers to the reproducibility of a test result (e.g. within 
How Reliable is Your Covid 19 Tests? Need to Validate and Verify Covid 19 Tests in the Laboratory Before Clinical Use

technologist, between technologist, within run and between run) repeatability. Clinical sensitivity refers to the ability of a test to detect a disease or clinical condition, while clinical specificity refers to the degree to which a test is negative when disease is absent.

Diagnostic sensitivity and specificity must be determined related to some "gold standard" (e.g. culture or composites clinical findings). The sensitivity of an assay equals [TP/(TP+ FN)] x 100 and the specificity of an assay equals $[\mathrm{TN} /(\mathrm{TN}+\mathrm{FP})](\mathrm{TP}=$ true positive, $\mathrm{TN}=$ true negative, $\mathrm{FN}=$ false negative, $\mathrm{FP}=$ false positive). Determination of sensitivity and specificity should be done in a blinded fashion (i.e. without prior knowledge of the patient's disease status).

Table summarises the performance characteristic that should be considered when planning method validation and method verification for both quantitative and qualitative methods.

\begin{tabular}{|l|c|c|c|c|}
\hline \multirow{2}{*}{ Characteristics to be evaluated } & \multicolumn{2}{|c|}{ Validation } & \multicolumn{2}{c|}{ Verification } \\
\cline { 2 - 4 } & Quantitative & Qualitative & Quantitative & Qualitative \\
\hline Limit of detection & Yes & No & Yes & No \\
\hline Sensitivity & Yes & Yes & Yes & Yes \\
\hline Selectivity & Yes & Yes & Yes & No \\
\hline Linearity & Yes & No & Yes & No \\
\hline Measuring interval & Yes & No & Yes & Yes \\
\hline Matrix effect & Yes & Yes & Yes & Yes \\
\hline Trueness, bias & Yes & Yes & Yes & Yes \\
\hline Precision (repeatability and reproducibility) & Yes & Yes & Yes & Yes \\
\hline Accuracy & Yes & Yes & Yes \\
\hline
\end{tabular}

\section{How to demonstrate}

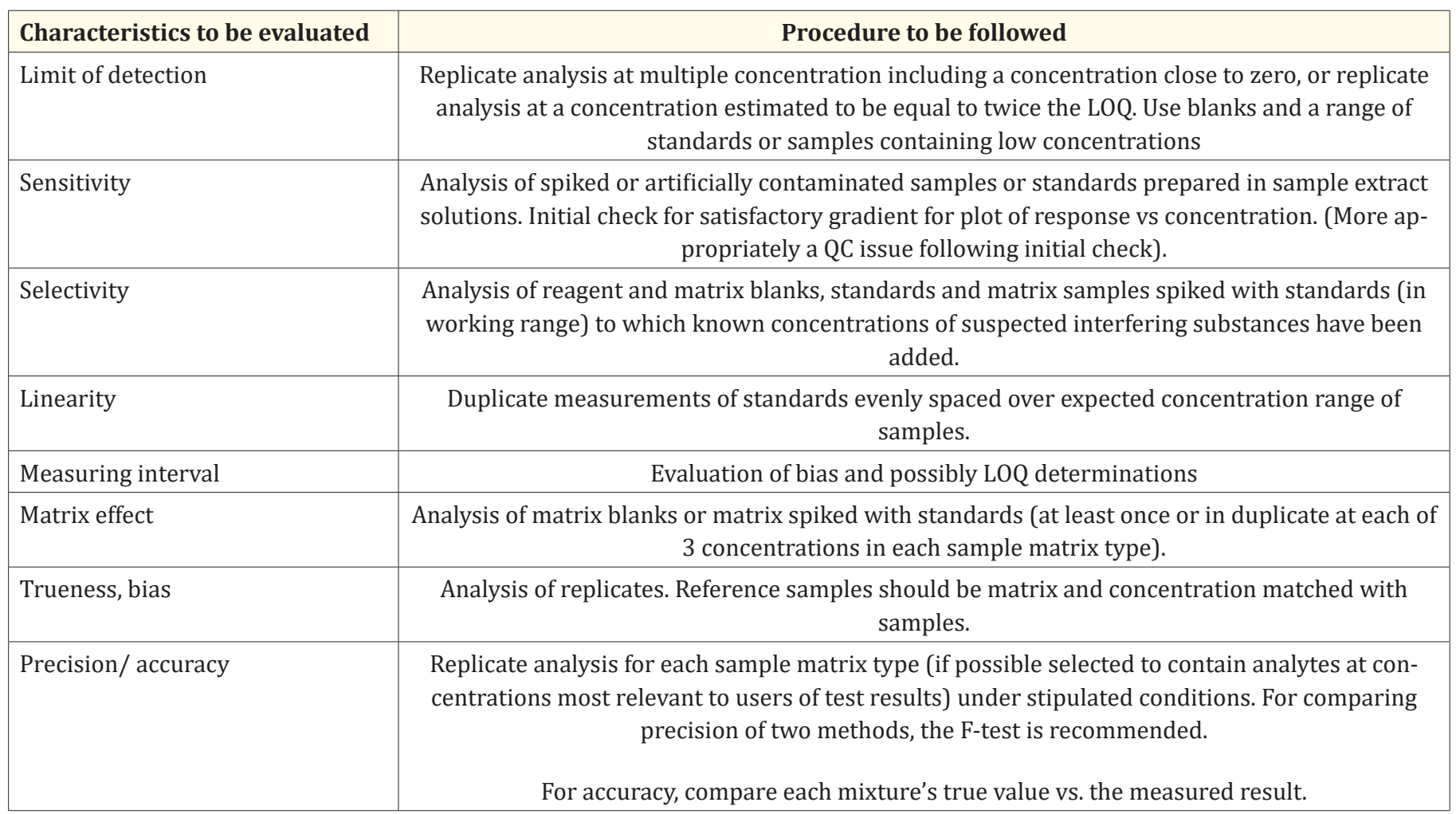


Not all parameters need to be assessed for all methods. The rigour of validation should be sufficient to ensure that test results produced by a method are technically sound and will satisfy the client's needs. Well planned method validation studies will be based on a clear understanding of the specific requirements for the method in use. Within this framework, carefully designed experiments will provide information to satisfy more than one of the parameters [7].

Till as approved External Quality Assurance Scheme (EQAS) becomes available, laboratories approved by regulatory authorities to perform Covid 19 diagnostic tests must participate in inter laboratory comparison with established laboratories or those designated by ICMR as Reference Laboratories by sending atleast five samples (three positive and two negatives) of their RT PCR results and starting clinical sample testing and processing only after obtaining $100 \%$ match.

As serology tests become available, USA regulators have instructed the manufacturers to test atleast 30 serum samples from RT PCR confirmed Covid 19 patients and atleast 80 samples collected in pre Covid 19 days (or antibody negative samples of which atleast 10 must be HIV positive). Acceptance criteria for issue of EUA is: 1 . Total antibody test $>90 \%$ PPA and 95\% NPA; 2 . For IgM specific test $>70 \%$ of PPA; for IgG specific test $>90 \%$ PPA with no cross reaction with HIV positive samples.

Individual laboratories are encouraged to: 1 . Choose a test with high specificity (> 99\%); 2 . Test individuals with high pre-test probability of being positive and 3. Apply orthogonal testing algorithm in which persons who initially test positive are tested with a second test. Effective orthogonal algorithms are generally based on testing a patient sample with two tests, each with unique design characteristics (e.g. antigens $\mathrm{N}$ followed by $\mathrm{S}$ or format ELISA and CLIA) [8].

USA FDA has placed important performance parameters of some of these serological tests on their website (Table).

\section{Orthogonal algorithm for serology testing}

First Pan Ig are detected against the most immunogenic antigen in the virus, $\mathrm{NC}$, all positive samples are tested for presence of antibodies to Spike protein. Spike is used by the virus to bind to ACE 2 receptors on human cells and cause infection. Anti Spike antibodies have been shown to be viral neutralizing and have been shown to protect animals from infection or reinfection.

\begin{tabular}{|l|c|c|c|c|c|c|c|}
\hline $\begin{array}{l}\text { Company Instrument } \\
\text { Attribute }\end{array}$ & $\begin{array}{c}\text { Abbott } \\
\text { Architect }\end{array}$ & $\begin{array}{c}\text { Roche } \\
\text { Elecsys }\end{array}$ & $\begin{array}{c}\text { Diasorin } \\
\text { Liasion }\end{array}$ & $\begin{array}{c}\text { Euro } \\
\text { immune }\end{array}$ & Ortho & Siemens & $\begin{array}{c}\text { Biorad } \\
\text { Platelia }\end{array}$ \\
\hline Antibody detected & IgG & Pan Ig & IgG & IgG & IgG & Pan Ig & Pan Ig \\
\hline Target & NC & NC & Spike & Spike & Spike & Spike & NC \\
\hline Method & CMIA & ECLIA & CMIA & ELISA & CLIA & CLIA & ELISA \\
\hline Sensitivity & 100 & 100 & 97.6 & 90 & 90 & 100 & 92.2 \\
\hline Specificity & 99.6 & 99.8 & 99.3 & 100 & 100 & 99.8 & 99.6 \\
\hline PPA at 5\% & 92.9 & 96.5 & 88 & 100 & 100 & 96.5 & 91.7 \\
\hline NPA at 5\% & 100 & 100 & 98.9 & 99.5 & 99.5 & 100 & 99.6 \\
\hline NC = Nucleocapsid (the most immunogenic antigen in SARS CoV 2) & & & & \\
\hline
\end{tabular}

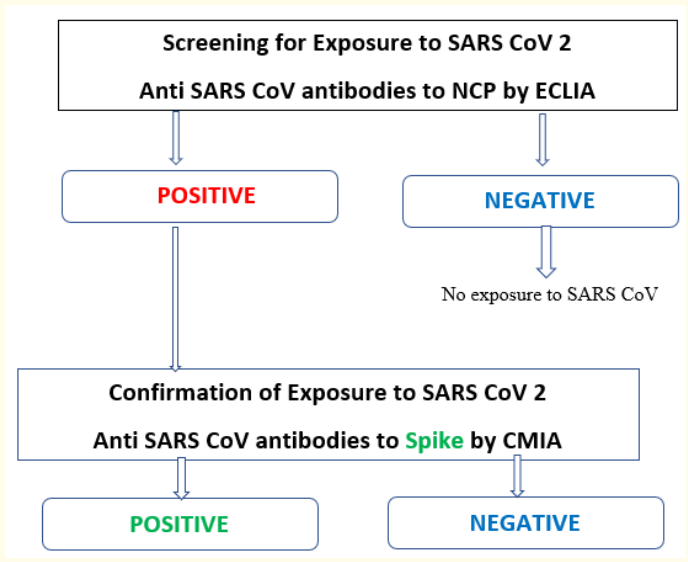

Figure

\section{Conclusion}

Table summarises the performance characteristic that should be considered when planning method validation and method verification for both quantitative and qualitative methods.

Not all parameters need to be assessed for all methods. The rigour of validation should be sufficient to ensure that test results produced by a method are technically sound and will satisfy the client's needs. Well planned method validation studies will be based on a clear understanding of the specific requirements for the method in use. Within this framework, carefully designed experiments will provide information to satisfy more than one of the parameters 


\section{Bibliography}

1. Chan JFW., et al. "Improved molecular diagnosis of Covid 19 by the novel, highly sensitive and specific Covid $19 \mathrm{RdRp} / \mathrm{Hel}$ Real time reverse transcription PCR validated in vitro and with clinical specimens". Journal of Clinical Microbiology 58 (2020): e00310-e00320.

2. Corman VM., et al. "Detection of 2019 novel coronavirus (2019 nCoV) by real time RT PCR". Euro Surveill 25.3 (2020): 2000045.

3. ICMR Press Release: How India ramped up Covid 19 testing capacity (2020).

4. ISO Medical Laboratories - Requirement for quality and competence (2012).

5. Jennings L., et al. "Recommended principles and practices for validating clinical molecular pathology tests". Archives of Pathology and Laboratory Medicine 133 (2009): 743-755.

6. USA FDA: Molecular Diagnostics Template for Laboratories. Policies for Coranavirus Disease 2019 Tests during the Public Health Emergency (2020): 1-9.

7. Guidelines for the validation and verification of quantitative and qualitative test methods". Technical Note (2012): 1-32.

8. CDC. Atlanta: Interim Guidelines for COVID 19 antibody testing (2020).

\section{Assets from publication with us}

- Prompt Acknowledgement after receiving the article

- Thorough Double blinded peer review

- Rapid Publication

- Issue of Publication Certificate

- High visibility of your Published work

Website: https://www.actascientific.com/

Submit Article: https://www.actascientific.com/submission.php

Email us: editor@actascientific.com

Contact us: +919182824667 